\title{
Min-Cut Based Partitioning for Urban LTE Cell Site Planning
}

\author{
Alexander Engels, Michael Reyer, Andreas Steiger, and Rudolf Mathar \\ Institute for Theoretical Information Technology \\ RWTH Aachen University \\ D-52056 Aachen, Germany \\ Email: \{engels, reyer, mathar\}@ti.rwth-aachen.de, andreas.steiger@rwth-aachen.de
}

\begin{abstract}
Common approaches for partitioning cell site planning problems into smaller instances typically suffer from the lack of knowledge about the number of clusters $k$ that is appropriate for the particular problem. When applying an iterative processing over methods such as the $k$-means algorithm or the related $k$-medoids approach to determine the optimum $k$, the computational effort can be higher than solving the original problem instance directly. This is particularly the case if the optimum $k$ is small, which happens most likely in urban environments where the user density and the number of cells are high. In this paper, we propose a graph-based method using minimum cut operations to partition a planning instance automatically into an appropriate number of $k$ sub-problems. We demonstrate the benefits of this approach by numerical evaluation of exemplary application to the problem of planning LTE cell site locations in an urban environment.
\end{abstract}

Index Terms-LTE network planning, graph theory, multiobjective optimization, interference modeling.

\section{INTRODUCTION}

Since recent studies predict a boost of mobile data traffic of more than one magnitude within the next four years [1], operators prepare their networks to cope with the future demand. Most operators introduce fourth generation (4G) wireless communication systems based on the Long-Term Evolution / System Architecture Evolution (LTE / SAE) standard [2]. This means that there is a continuous need for LTE cell site planning, i.e., deployment and configuration of eNodeBs. Particularly for urban environments, where the cell density has to be quite high, this is a very resource consuming task that suffers from a high computational complexity. Generally, inter-cell interference causes strong interdependence between potential sites and configurations, respectively. This makes an optimal selection a hard combinatorial problem that, moreover, is of multi-objective nature. Typical Key Performance Indicators (KPIs) that are combined into a joint objective are coverage, capacity, and economical aspects [3], [4].

One approach to reduce computational complexity is the partitioning of the planning problem into smaller instances, i.e., the spatial decomposition of relevant elements into separately treated clusters. Common methods for clustering of multidimensional data sets are, for instance, the $k$-means algorithm and the related $k$-medoids approach [5], [6]. Both methods aim at finding clusters with minimal intra-cluster distortion for a prespecified number of clusters $k$. While the points representing the clusters in the $k$-means algorithm are centroids that are not necessarily elements from the original data set, the $k$-medoids method considers medoids as cluster representatives, i.e., it operates only on initially existing elements. The latter approach is particularly preferred if the computation of distances from existing points to new points is expensive. In the context of wireless communications, this is the case if signal strength between transmitter and receiver points is not computed on basis of a distance related (semi-empirical) path loss model but with respect to more accurate models, e.g., ray optical methods [7], [8]. Such methods are utilized preferably for urban environments, where physical propagation effects at densely deployed buildings cause a very diverse path loss profile. Figure 1 illustrates this situation exemplarily for an artificially deployed transmitter. Both clustering approaches, however, do not automatically determine the suitable number of clusters $k$ for the considered scenario. One way to obtain an appropriate $k$ is the iterative evaluation of problem partitioning, i.e., to start with a large $k$, to compute optimal solutions for the corresponding clustering, and to stop when the objective function is not improved by using a smaller $k$. The major drawback of this method is that for each iteration step over $k$, the optimization problem has to be solved for all clusters. If the optimum $k$ is small, the overall computational effort can be larger than solving the original problem instance directly.

In this paper, we propose a minimum cut (min-cut) based approach for problem partitioning that automatically finds an appropriate number of clusters $k$ without solving any planning problems in between. Furthermore, our method does not rely on a random selection of initial cluster center points but arranges clusters automatically around their native centers. We apply the proposed method exemplarily to the problem of planning LTE cell site locations in an urban environment and demonstrate its benefits by numerical evaluation.

The rest of this paper is organized as follows. In Section II, we discuss related work before we introduce the considered system model in Section III and describe the cell site planning tasks in Section IV. We discuss the problem of partitioning planning instances into smaller sub-problems and propose our approach in Section V. In Section VI, we apply our method exemplarily and evaluate benefits numerically. Finally, we conclude this paper and discuss future work in Section VII. 


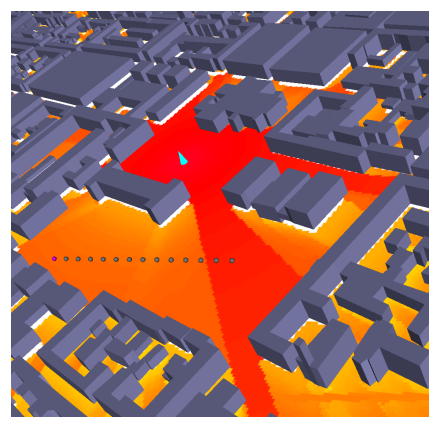

Figure 1. Diverse signal strength distribution in an urban environment.

\section{RELATED WORK}

In [9] and [10], Yu et al. apply a $k$-means clustering method to partition instances for planning $802.16 \mathrm{j}$ base station and relay station locations into smaller sub-problems. Since the considered path loss models are semi-empirical, computation of the distance measure to new points, i.e., cluster centroids, is simple. After reassignment of transmitter-to-receiver links for the overall deployment solution - obtained by combining the sub-problem solutions - they achieve results very close to the optimum, while the clustering approach saves up to $70 \%$ of computation time even for small $k$. The number of clusters, however, is chosen manually with respect to the considered planning scenario. In [11], Partitioning Around Medoids (PAM) - a variant of the $k$-medoids method - is combined with a modified Density-Based Spatial Clustering of Applications with Noise (DBSCAN) [12] to partition mobile network planning problems. The number of clusters $k$ is initially set to the expected number of required cells concerning coverage and capacity requirements. The number is iteratively increased according to a density-based criterion whenever the demand in a cluster requires deployment of a second base station, i.e., the computed number of clusters equals the number of deployed base stations. This approach is very sensitive to the initial estimation of potentially required $k$ and might suffer from the risk of "over-clustering" which can lead to neglection of inter-cell dependencies such as interference.

$\mathrm{Su}$ et al. introduce a graph-based representation of multi-cell and multi-user wireless networks in [13]. This structure is used to identify base stations that have to coordinate themselves for simultaneous downlink transmission with respect to intercell interference. Although our purposes are different from this context, we utilize a similar graph-based representation to apply min-cut algorithms for problem partitioning. Clustering based on minimum-cut trees is a very popular technique in image segmentation, biology, or general network analysis [14]. The basic idea is to partition the initial data set hierarchically by applying min-cut operations such that the resulting clusters have small inter-cluster dependencies and relatively large intracluster dependencies [15]. In [16], cut expansion and cut conductance are introduced as useful measures to describe cluster quality. In our work, we use a modified version of the conductance as quality measure.

\section{System MOdEL}

We consider planning of LTE cell sites in an urban environment, i.e., where to deploy eNodeBs (eNBs) such that given user demands can be supported by downlink transmission in the radio network. Generally, LTE cells utilize OFDMA as access technology to serve users (UEs) in a shared frequency spectrum. According to the LTE system specification [2], we distinguish 16 Channel Quality Indicators (CQI) to describe link quality from eNBs to users. Each CQI corresponds to a supported modulation scheme and code rate for downlink transmission with respect to a fixed bit error rate, i.e., we can compute the spectral efficiency in terms of bits per second per Hertz for each CQI. The lowest non-zero spectral efficiency is $0.25[\mathrm{bps} / \mathrm{Hz}]$ for QPSK and code rate $1 / 8$ and the largest spectral efficiency is 4.8 [bps/Hz] for 64-QAM and code rate $4 / 5$. The system link budget specification defines what Received Signal Power (RSP) is required to support a minimum downlink throughput of $95 \%$ of the maximum possible at different CQIs. Different QoS requirements of an UE can be modeled by modifying the corresponding CQI specifications accordingly.

To compute link-wise RSP information, we utilize the ray optical approaches presented in [7] and [8], respectively. By comparing the achievable RSP to the CQI requirements, the maximum supportable CQI is selected and the corresponding spectral efficiency describes the maximum supported link quality. Since interference is one of the main limiting effects for network coverage, capacity, and performance, we introduce the following model to incorporate inter-cell interference.

\section{A. Interference Approximation Model}

Generally, resource allocation considering interference is performed on basis of the Signal-to-Interference plus Noise Ratio (SINR) that Physical Resource Block (PRB) experience for a certain UE-to-eNB assignment [17]. Taking into account the SINR, optimal resource allocation is a combinatorial problem that is computationally hard for practical problem sizes. Hence, we utilize a linear approximation model that partitions the consumed bandwidth into resource $b_{s}^{\mathrm{SRV}}$ utilized for transmission on basis of the Signal-to-Noise Ratio (SNR) and into resource $b_{s}^{\mathrm{ITF}}$ that is blocked for interference mitigation, i.e., the total consumed bandwidth is

$$
b_{s}^{\mathrm{SRV}}+b_{s}^{\mathrm{ITF}} .
$$

The blocked bandwidth is computed as linear combination over resources of interfered transmissions, where a link from $s^{\prime}$ to user $t$ is interfered by $s$ with impact factor $q_{s s^{\prime} t} \in[0,1]$. The impact factor basically depends on the signal strength ratio and the frequency reuse potential of related cells. Since the model describes the potential amount of bandwidth that has to be reserved in the spectrum to support the overall UE demand, we call it Bandwidth Reservation Concept (BRC). In particular, our approach models the expected average amount of bandwidth that is consumed according to resource allocation algorithms performed by an eNB. Note that it does not 


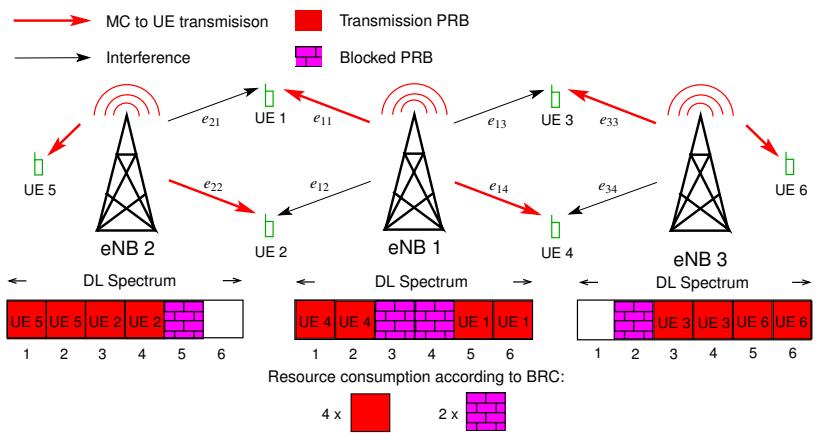

Figure 2. Exemplary PRB allocation at eNBs and estimated spectrum consumption for eNB 1 .

describe where the consumed resources are allocated in the spectrum and, hence, skips the combinatorial problem of PRB assignment.

Figure 2 exemplarily illustrates application of the approximation assuming equal signal power for all transmission and all interference links and that each eNB-to-UE transmission requires two PRBs to fulfill UE rate demand. The depicted allocation is obtained by assuming resource allocation algorithms that can take advantage of an average frequency reuse potential of $1 / 2$ for each eNB. For practical application, the reuse potential might be calibrated differently for inner cells and outer cells, i.e., cells with many and a few interfering neighbors, respectively.

\section{LTE CEll Site Planning}

Considering notation according to Table I, the cell site planning problem is the following: Which eNBs out of the set of candidates $\mathcal{S}$ should be deployed to serve a maximum of expected user rate demand at minimum costs. We apply the demand node concept from [18] to abstract from single UEs by aggregating a suitable number of UEs into one Demand Node $(D N)$. Hence, DNs model the spatial distribution of aggregated UEs as well as their cumulated rate demand on average.

Inspired by [19] and [20], we consider the optimization problem

$$
\max \left[\sum_{(s, t) \in \mathcal{S} * \mathcal{T}} z_{s t}\left(\lambda_{\text {basic }}+\lambda_{\text {rate }} r_{t}\right)-\sum_{s \in \mathcal{S}} c_{s} x_{s}\right]
$$

subject to

$$
\begin{array}{ll}
\sum_{s \in \mathcal{S}_{t}} z_{s t} \leq 1 & \forall t \in \mathcal{T} \\
z_{s t} \leq x_{s} & \forall(s, t) \in \mathcal{S} * \mathcal{T} \\
\sum_{t \in \mathcal{T}_{s}} \frac{r_{t}}{e_{s t}} z_{s t}+b_{s}^{\mathrm{ITF}} \leq B_{s}+\left(1-x_{s}\right) \cdot \infty & \forall s \in \mathcal{S} \\
b_{s}^{\mathrm{ITF}}=\sum_{\left(s^{\prime}, t\right) \in \mathcal{S} * \mathcal{T}, s^{\prime} \neq s} q_{s s^{\prime} t} \frac{r_{t}}{e_{s^{\prime} t}} z_{s^{\prime} t} & \forall s \in \mathcal{S}
\end{array}
$$

\begin{tabular}{|c|c|}
\hline Symbol \& domain & Parameter description \\
\hline $\mathcal{S}=\left\{1, \ldots, N_{S}\right\}$ & Index set of eNB candidates \\
\hline $\mathcal{T}=\left\{1, \ldots, N_{T}\right\}$ & Index set of demand nodes (DN) \\
\hline$s \in \mathcal{S}, t \in \mathcal{T}$ & Representative indices \\
\hline$c_{s} \in \mathbb{R}_{\geq 0}$ & eNB costs \\
\hline$r_{t} \in \mathbb{R}_{\geq 0}$ & DN data rate demand \\
\hline$B_{s} \in \mathbb{R}_{\geq 0}$ & Total bandwidth available at eNB \\
\hline$e_{s t} \in \mathbb{R}_{\geq 0}$ & $\begin{array}{l}\text { Supported spectral efficiency from eNB } \\
\text { to DN according to Sec. III }\end{array}$ \\
\hline$e_{\min } \in \mathbb{R}_{>0}$ & $\begin{array}{l}\text { Minimum spectral efficiency to enable } \\
\text { transmission (CQI 1) }\end{array}$ \\
\hline$\underline{q}_{s \underline{s}^{\prime}} \in[0,-1]$ & Interference impact factors \\
\hline Symbol \& domain & Variable description \\
\hline$x_{s} \in\{0,1\}$ & eNB deployment indicator \\
\hline$z_{s t} \in\{0,1\}$ & Coverage (assignment) indicator \\
\hline
\end{tabular}

Table I

INPUT PARAMETERS AND VARIABLES.

where the sets $\mathcal{S} * \mathcal{T}, \mathcal{S}_{t}$, and $\mathcal{T}_{s}$ are defined as

$\mathcal{S} * \mathcal{T}=\left\{(s, t) \in \mathcal{S} \times \mathcal{T} \mid e_{s t} \geq e_{\min }\right\}$,

$\mathcal{S}_{t}=\{s \in \mathcal{S} \mid(s, t) \in \mathcal{S} * \mathcal{T}\}, \mathcal{T}_{s}=\{t \in \mathcal{T} \mid(s, t) \in \mathcal{S} * \mathcal{T}\}$.

For appropriately chosen parameters $\lambda_{\text {basic }}$ and $\lambda_{\text {rate }}$, the objective leads to maximization of operator profit [20]. By (1) and (2), each DN $t$ can be covered by at most one deployed BS $s\left(z_{s t}=1\right)$. The rate demand $r_{t}$ of each covered DN is fully served. The corresponding bandwidth is reserved according to the first term of constraint (3). The amount of required bandwidth depends on the link quality, i.e., on the spectral efficiency $e_{s t}$ the link supports. The bandwidth $b_{s}^{\text {ITF }}$ that has to be blocked to mitigate inter-cell interference is computed according to the model from Section III-A as (4). For each deployed eNB, the overall consumed bandwidth has to stay below the available bandwidth $B_{s}$. The infinity term on the right hand side of (3) removes this constraint for all non-deployed eNBs to avoid feasibility problems.

Since objective function and constraints of the optimization problem are linear, it is an Integer Linear Program (ILP) [21] that can be solved by solvers such as CPLEX or Gurobi.

\section{Partitioning of Planning Problems}

The general processing for partitioning cell site planning problems is the following: The planning instance is separated into clusters that contain subsets of base station (BS) candidates and DNs. The corresponding sub-problems are solved independently and sub-solutions are aggregated to an overall solution by selecting deployed BSs from the clusters. Since inter-cluster interference is ignored, reassignment of DNs with respect to fixed BS deployment in the original planning scenario is necessary to obtain a reliable objective value. No matter what clustering approach is chosen, the distance measure between points is the key component that determines which points belong to the same cluster. In the context of wireless networks, a reliable distance measure has to reflect dependencies between points strongly related to signal quality. 


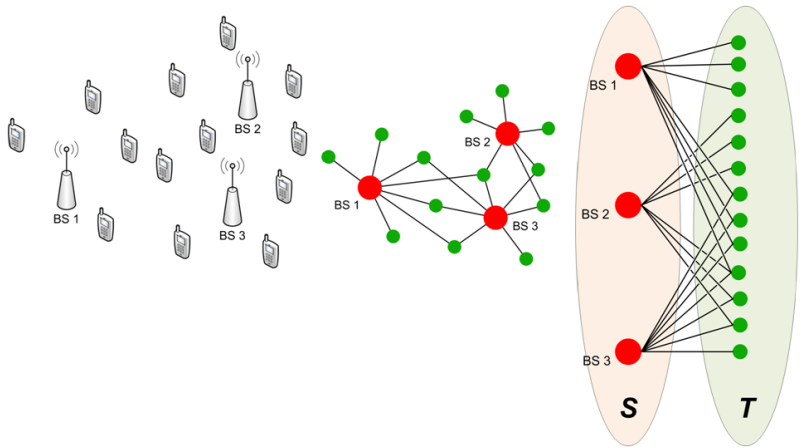

Figure 3. Graph-based representation of wireless networks.

\section{A. A graph-based distance measure}

As mentioned in Section I and illustrated by Figure 1, Euclidean distance or semi-empirical path loss are not the right measures to describe signal quality between points in an urban environment. Hence, we propose the following distance measure that is pre-computable between all relevant points of the planning problem. According to Figure 3, we describe a cell planning instance - or a wireless network scenario as bipartite graph $G=(S, T, E)$, where $S$ contains all BS (eNB) candidates, $T$ the DNs (UEs), and the weights $w_{i j}$ of undirected edges $E$ are equal to the spectral efficiency (alternatively RSP) on the corresponding links and are computed by a ray optical approach according to Section III. A suitable distance measure for clustering is then given by the cost of the shortest path [21] between nodes in $G$, where the cost function for an edge is the reciprocal of its weight, i.e., $1 / w_{i j}$.

\section{B. Min-cut based partitioning}

Considering the graph-based representation of a cell site planning instance as described above, we propose a hierarchical approach [6] to partition the graph $G$ into clusters that have a strong intra-cluster and a weak inter-cluster relationship. The strength of inter-cluster relationship between two clusters is quantified by the cost of the cut that separates the disjoint partitions $P, \bar{P} \subset G$. Similarly, the strength of intra-cluster relationship is indicated by the sum over all edge weights in the cluster. To find an appropriate clustering criterion, we consider the conductance [16] of a cut $(P, \bar{P})$

$$
\phi(P, \bar{P})=\frac{\sum_{i \in P, j \in \bar{P}} w_{i j}}{\min \{w(P), w(\bar{P})\}},
$$

where $w(P)$ is the sum over all edge weights in partition (cluster) $P$. Roughly speaking, a cut with low conductance provides a good trade-off between cost for decomposition and cluster quality. It turned out that the sum over edge weights can become fairly large if DNs are located in line-of-sight positions and that, hence, even well assessed clusterings can be very unbalanced. Therefore, conductance is not sufficient to achieve a beneficial partitioning for cell site planning problems. We extend measure (5) by considering partition balance and the BS-to-sum-rate ratio in clusters as multiplicative terms, i.e.,

$$
\Phi(P, \bar{P})=\phi(P, \bar{P}) \cdot \frac{\max \{|P|,|\bar{P}|\}}{\min \{|P|,|\bar{P}|\}} \cdot \max \left\{\frac{\left|S_{P}\right|}{R_{P}}, \frac{\left|S_{\bar{P}}\right|}{R_{\bar{P}}}\right\}
$$

serves as trade-off measure to assess a cut $(P, \bar{P})$, where $R_{P}$ is the DN sum rate in a partition $P$; when considering equal rate demand for all DNs, the sum rate might be replaced by DN cardinality $\left|T_{P}\right|$. Finding a cut that minimizes (6) is computationally hard since the number of possible cuts grows exponentially with the number of nodes in $G$. Therefore, we apply the following heuristic to shrink the number of cut candidates: First, the considered graph is extended by an artificial source node $q$ that is connected to all BS nodes $s \in S$, where each edge from $q$ to $s$ is weighted slightly larger than the sum over all further edges of $s$, e.g.,

$$
w_{q s}=\sum_{t \in T} w_{s t}+1 \text {. }
$$

Second, we successively compute the minimum cuts (min-cuts) from $q$ to each node $t \in T$, where all edges to a respective sink node $t$ temporarily get an infinite weight. Adapting edge weights of source and sink node in the described way prevents from finding trivial cuts that simply separate one of these nodes. Algorithms to compute min-cuts are computationally efficient, e.g., by solving the max-flow problem [21]. From the set of min-cuts over $T$ we select the one for which (6) is minimal and consider the corresponding two partitions as cluster candidates with achieved trade-off measure $\Phi$. Whether the candidates are accepted as suitable clustering is decided by a criterion that takes into account $\Phi$ and the trade-off of previous partitioning. Algorithm 1 describes the overall procedure for hierarchical partitioning of $G$ utilizing min-cut based clustering as described above. By choosing a degrada-

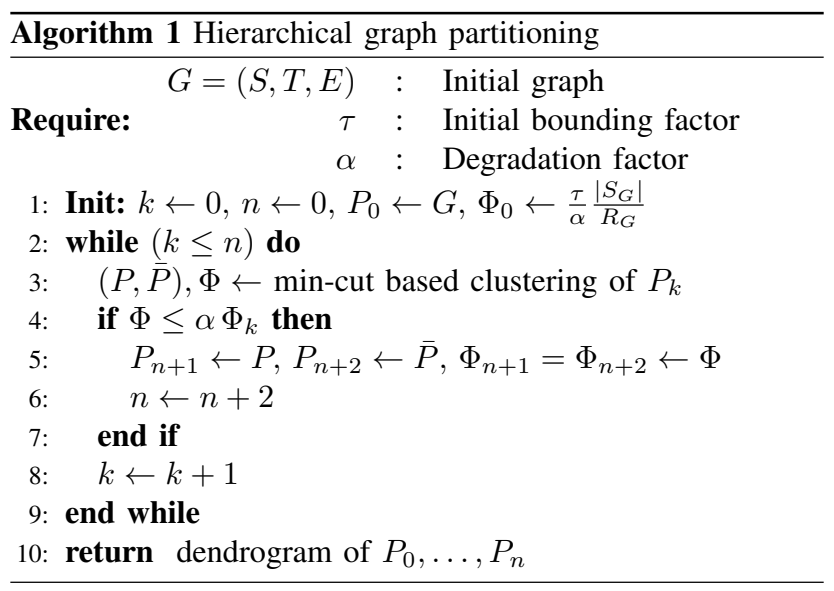

tion factor $\alpha \leq 1$, we ensure that a subsequent partitioning is applied only if it keeps or improves its parent cluster trade-off measure. The decision might be complemented by requiring a minimum number of BSs and DNs in the sub-clusters. The initial trade-off measure $\Phi_{0}$ in line 1 is derived on basis of the bounding factor $\tau$ that reflects the worst case constellation 
of conductance and partition balance that is still tolerable for partitioning $G$. The leaf nodes of the returned dendrogram describe the partitioning of $G$, see Figure 4 (d).

For subsequent partitioning, conductance tends to increase and partitions get more balanced. The BS-to-sum-rate ratio does not vary that much but provides a valuable criterion in split decision situations. The number of clusters $k$ equals the number of dendrogram leaf nodes and is implicitly determined by the evolution of hierarchical partitioning trade-offs.

\section{Numerical Evaluation}

To demonstrate performance and benefits of our approach, we consider the following setup for planning LTE cell sites in an urban environment: Figure 4 (a) shows the relevant city area and 60 potential locations for eNB deployment (red dots). Each eNB operates with $43 \mathrm{dBm}$ average transmit power and provides $B=5 \mathrm{MHz}$ DL transmission bandwidth at a center frequency of $2 \mathrm{GHz}$. While typical cell site planning might cover multiple configurations for eNBs, we simply consider average transmission power and one antenna pattern (omnidirectional, $0^{\circ}$ downtilt). We assume operational expenses of $1800 €$ per month covering site rental, leased line rental, air-conditioning, and maintenance [4]. Furthermore, we choose $\lambda_{\text {basic }}=50 €$ and $\lambda_{\text {rate }}=0.5 € /$ kbps to describe the monthly fee paid to the operator. Resulting from an aggregation of service-related average rates, we consider a constant rate demand of $320 \mathrm{kbps}$ per DN. We compute the maximum supported spectral efficiency for all potential eNBto-DN links according to Section III and assume interference impact factors to scale with the ratio of related link qualities. All computations were executed on a $2.2 \mathrm{GHz}$ Intel Core i7 architecture with $8 \mathrm{~GB}$ RAM and using Gurobi Optimizer version 5.0 [22] for optimization.

Since we want to evaluate our partitioning approach particularly for planning scenarios that actually have a clustered structure, we carry out a 2-dimensional Gaussian sampling [6] on a set of quasi-uniformly distributed DNs. Figure 4 (b) depicts such a sampling for a random choice of 5 Gaussian distributions, where crosses visualize the distribution means. After applying our min-cut based partitioning to the original planning instance, we solve the planning problem for all subinstances and obtain the overall eNB deployment set as union over the sub deployment decisions. To keep comparison to results from solving the original problem fair, we perform a final reassignment of eNB-to-DN links by solving the initial instance for the fixed eNB deployment solution: All comparison values are taken from the corresponding result.

We consider time savings and solution quality as relevant assessment criteria, both with respect to the optimal solution obtained for the original planning instance. Table II shows the evaluation results for three different cluster sampling sizes carried out on the same planning scenario. The number of sampled clusters is positively correlated with the number of DNs and, hence, positively correlated with the (average) computation time that is required to solve the planning instance optimally. For each sampling size, we generated five planning
Table II

EVALUATION RESULTS.

\begin{tabular}{|l|l|l|l|l|}
\hline $\begin{array}{l}\text { No. of } \\
\text { sampled } \\
\text { clusters }\end{array}$ & $\begin{array}{l}\text { Average } \\
\text { no. of } \\
\text { DNs }\end{array}$ & $\begin{array}{l}\text { Average } \\
\text { computation } \\
\text { time [s] }\end{array}$ & $\begin{array}{l}\text { Computation } \\
\text { time with } \\
\text { partitioning }\end{array}$ & $\begin{array}{l}\text { Solution } \\
\text { quality with } \\
\text { partitioning }\end{array}$ \\
\hline 3 & 194 & 1229 & $21 \%(1.6 \%)$ & $95 \%(94 \%)$ \\
4 & 272 & 3755 & $9 \%$ & $96.8 \%$ \\
5 & 325 & 9690 & $22 \%(2.4 \%)$ & $99.3 \%(99 \%)$ \\
\hline
\end{tabular}

scenarios at random and applied Algorithm 1 for $\alpha=1$ and initial conductance-balance bounding factor $\tau=0.75 \cdot 2=1.5$. Figure 4 (d) shows the algorithm results for this parameter setting and exemplary application. The depicted numbers describe the trade-off measure $\Phi$ on the corresponding partitioning level and partitioning stops (red cross) when the tradeoff measure starts to increase.

Table II demonstrates that we achieve significant savings of computation time while keeping at least $95 \%$ solution quality. For a number of five sampled clusters, we observe an even higher accuracy of over $99 \%$. This might be explained by the fact that inaccuracies due to partitioning have higher potential to cancel out in larger planning scenarios. We obtain a planning instance with three sampled clusters that is not partitioned and another unpartitioned one for five sampled clusters. Excluding those instances from the evaluation examples leads to the results written in brackets: While solution quality degrades only marginally, removing the corresponding computation times affects time savings significantly. The best solution quality that we observe in the latter case is $100 \%$, associated with $7 \%$ of original computation time - which is the worst time saving. The average number of optimally deployed eNBs increases from 5 to 7 and 9 over the number of sampled clusters. Applying the partitioning approach leads on average to one more deployed eNB in cell planning solutions.

\section{A. The wrong choice of $k$}

Due to the diversity in urban radio wave propagation, it is not obvious which points potentially form a cluster and, hence, how many clusters exist in an urban environment. This is illustrated, for instance, by the clustering results depicted in Figure 4 (c): The lower right (yellow) cluster is connected to points that are located in the upper left part of the scenario as well as to points in the middle left part. Comparing those connections to the free space (white) areas of the city indicates that this effect is strongly related to the path loss characteristics amongst involved points. Thus, it is difficult to determine an appropriate number of clusters $k$ even by human decision.

To quantify the impact of choosing an inappropriate $k$, we exemplarily took the two clusters from above that are not partitioned and forced a partitioning into $k=4$ clusters. For both examples, the related solution quality degraded to $91 \%$. While in both solutions too many eNBs were deployed due to the fact that candidates from other artificial clusters were not "visible", interference effects additionally affected the overall solution negatively for the larger planning example. 


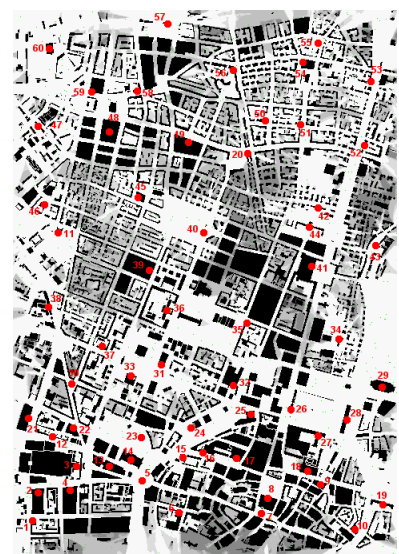

(a) Cell site planning scenario



(b) Clustered DN distribution.

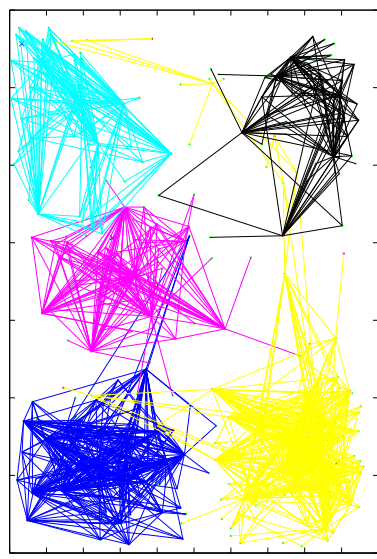

(c) Min-cut based partitioning.

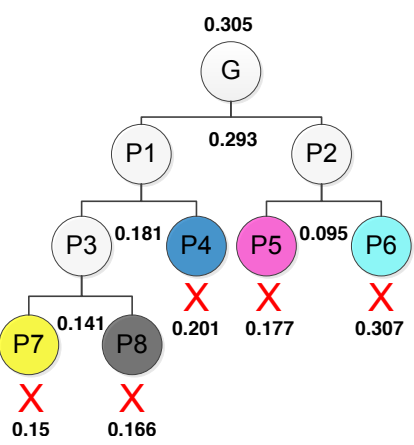

(d) Dendrogram for $\alpha=1, \tau=1.5$.

Figure 4. Exemplary planning scenario considering 60 eNB deployment candidates in Munich city (building data from [23]).

\section{CONCLUSiOnS AND Future WORK}

In this work, we proposed an approach for min-cut based partitioning of cell site planning problems in urban environments. Compared to common clustering methods such as $k$ means clustering or PAM, our approach does not include any random components and does not require a priori knowledge of intended number of clusters $k$. In fact, our approach automatically finds a beneficial number of partitions according to a natural clustering criterion that evolutionary evolves in a hierarchical manner. Application of our approach to the problem of LTE cell site planning in an urban environment demonstrates its effectiveness in terms of solution accuracy and its potential to yield significant time savings. Such savings are essential for solving practical planning problems that grow exponentially with the instance size, e.g., if multiple configurations are considered for each eNB deployment candidate.

Future work particularly covers the adaption of the proposed partitioning approach to automatically determine cooperation clusters in the operating network, which allows for optimal configuration of cluster eNBs independently from eNBs of other clusters.

\section{REFERENCES}

[1] Cisco Systems, Inc., "Cisco visual networking index: Global mobile data traffic forecast update, 2011-2016," Feb. 2012. [Online]. Available: www.cisco.com

[2] E. Dahlman, S. Parkvall, J. Sköld, and P. Beming, $3 G$ Evolution: HSPA and LTE for Mobile Broadband, 2nd ed. Academic Press, 2008.

[3] E. Amaldi, A. Capone, and F. Malucelli, "Radio planning and coverage optimization of 3G cellular networks," Wirel. Netw., vol. 14, pp. 435447, 2008

[4] ECOSYS Deliverable 19, "Final techno-economic results on mobile services and technologies beyond 3G," Sep. 2006. [Online]. Available: www.optcomm.di.uoa.gr/ecosys/

[5] C. M. Bishop, Pattern Recognition and Machine Learning. New York, NY, USA: Springer, 2006.

[6] R. O. Duda, P. E. Hart, and D. G. Stork, Pattern Classification. New York, NY, USA: John Wiley \& Sons, 2001.

[7] R. Mathar, M. Reyer, and M. Schmeink, "A cube oriented ray launching algorithm for 3D urban field strength prediction," in Proceedings: IEEE International Conference on Communications (ICC), Glasgow, Scotland, UK, Jun. 2007, pp. 5034-5039.
[8] F. Schröder, M. Reyer, and R. Mathar, "Efficient implementation and evaluation of parallel radio wave propagation," in EuCAP 2011 - 5th European Conference on Antennas and Propagation, Apr. 2011, pp. $2466-2470$

[9] Y. Yu, S. Murphy, and L. Murphy, "A clustering approach to planning base station and relay station locations in IEEE 802.16j multi-hop relay networks," in IEEE International Conference on Communications 2008. ICC '08., May 2008, pp. 2586-2591.

[10] Y. Zhang, WiMAX Network Planning and Optimization. Auerbach Publications, 2009.

[11] L. F. Ibrahim and H. A. Salman, "Using hyper clustering algorithms in mobile network planning," American Journal of Applied Sciences, vol. 8, pp. 1004-1013, Aug. 2011.

[12] M. Ester, H. P. Kriegel, and X Xu, "A density-based algorithm for discovering clusters in large spatial databases with noise," in Proceedings of 2nd International Conference on Knowledge Discovery and Data Mining (KDD-96). AAAI Press, 1996, pp. 226-231.

[13] G. Su, L. Cuthbert, and T. Zhang, "A graph-based dynamic coordinated clustering scheme for base station cooperation," in IET International Conference on Communication Technology and Application (ICCTA 2011), Oct. 2011, pp. 201-206.

[14] R. Görke, T. Hartmann, and D. Wagner, "Dynamic graph clustering using minimum-cut trees," in Algorithms and Data Structures, ser. Lecture Notes in Computer Science. Springer Berlin / Heidelberg, 2009, vol. 5664, pp. 339-350.

[15] Z. Wu and R. Leahy, "An optimal graph theoretic approach to data clustering: theory and its application to image segmentation," IEEE Transactions on Pattern Analysis and Machine Intelligence, vol. 15, no. 11 , pp. 1101-1113, Nov. 1993.

[16] G. Flake, R. Tarjan, and K. Tsioutsiouliklis, "Clustering methods based on minimum-cut trees," 2002.

[17] D. Tse and P. Visvanath, Fundamentals of Wireless Communication. Cambridge University Press, 2008.

[18] K. Tutschku, "Demand-based radio network planning of cellular mobile communication systems," in IEEE INFOCOM '98, vol. 3, Mar-Apr 1998, pp. 1054-1061.

[19] G. Claßen, A. M. C. A. Koster, and A. Schmeink. (2011) Planning wireless networks with demand uncertainty using robust optimization.

[20] A. Engels, M. Reyer, and R. Mathar, "Profit-oriented combination of multiple objectives for planning and configuration of 4G multi-hop relay networks," in International Symposium on Wireless Communication Systems (ISWCS), Sep. 2010, pp. 330-334.

[21] A. Koster and X. Muñoz, Graphs and Algorithms in Communication Networks. Springer Berlin Heidelberg, 2010.

[22] Gurobi Optimization, Inc. Gurobi optimizer 5.0. [Online]. Available: http://www.gurobi.com/products/gurobi-optimizer

[23] E. Damosso, COST Action 231: Digital mobile radio towards future generation systems, Final Report. Office for Official Publications of the European Communities, 1999. 